\title{
Letters
}

\section{Pediatric cannabis use and cardiac complications}

We read with interest the study by Ladha and colleagues, describing young adults (aged 18-44 yr) with myocardial infarctions (MI) and recent cannabis use; smoking was the primary route of consumption. ${ }^{1}$ Unfortunately, the findings described in this paper are not limited to adults. We recently reported on a 16-yearold adolescent who presented to our pediatric institution for chest pain after smoking cannabis, with an electrocardiogram revealing diffuse ST-segment elevations and echocardiogram showing global left ventricular dysfunction. ${ }^{2}$ Comprehensive toxicology testing showed only a $\Delta$-9tetrahydrocannabinol (THC) metabolite and was negative for cocaine and several other synthetic cannabinoids; an extensive infectious workup was unremarkable, making myocarditis unlikely. No focal coronary lesions or obstruction were detected with coronary angiography and a right ventricular septal endomyocardial biopsy obtained 36 hours after chest pain onset showing a subendocardial acute MI.

Cardiac complications of pediatric cannabis exposure described in the literature include both tachycardia and bradycardia, or bradycardia and hypotension, with the latter occurring typically in the setting of respiratory depression. ${ }^{3,4}$ Further, an 11-month-old died after cannabis exposure, with myocarditis diagnosed at autopsy. ${ }^{5}$ As cannabis use becomes more liberalized, pediatric exposures have increased. ${ }^{6}$ In 2020, the American Heart Association released a scientific statement summarizing the cardiovascular implications of cannabis: cardiomyopathy, MI, arrhythmias or sudden death, and cerebral vascular accidents are described. ${ }^{7}$ While THC stimulates the sympathetic nervous system with subsequent increased heart rate, myocardial oxygen demand, supine blood pressure and platelet activation in general, smoking THC can additionally increase carboxyhemoglobin concentrations 5-fold with endothelial dysfunction; moreover, increased lipoprotein oxidation and impaired oxygen binding have also been associated with negative cardiac outcomes. ${ }^{7}$ Education from a public health perspective targeted to the pediatric population is important to minimize these potential marijuana-related cardiac complications, both in terms of unintentional exposures and adolescent inhalational use. ${ }^{3}$

\section{Michele M. Burns MD MPH}

Staff physician, Harvard Medical

Toxicology Program, Division of

Emergency Medicine, Boston Children's

Hospital, Boston, Mass.

\section{Michael S. Toce MD MS}

Staff physician, Harvard Medical

Toxicology Program, Division of

Emergency Medicine, Boston Children's

Hospital, Boston, Mass.
Cite as: CMAJ 2021 November 15;193:

E1737. doi: $10.1503 / \mathrm{cmaj} .80288$

\section{References}

1. Ladha KS, Mistry N, Wijeysundera DN, et al. Recent cannabis use and myocardial infarction in young adults: a cross-sectional study. CMAJ 2021;193:E1377-84.

2. Toce MS, Farias M, Powell AJ, et al. Myocardial infarct after marijuana inhalation in a 16-yearold adolescent boy. Pediatr Dev Pathol 2019;22 1320-1.

3. Amirshahi MM, Moss MJ, Smith SW, et al. ACMT position statement: addressing pediatric cannabis exposure. J Med Toxicol 2019;15:212-4.

4. Wang GS, Le Lait MC, Deakyne SJ, et al. Unintentional pediatric exposures to marijuana in Colorado, 2009-2015. JAMA Pediatri 2016;170:1-6.

5. Nappe TM, Hoyte CO. Pediatric death due to myocarditis after exposure to cannabis. Clin Pract Cases Emerg Med 2017;1:166-70.

6. Whitehill JM, Harrington C, Lang C, et al. Incidence of pediatric cannabis exposure among children and teenagers aged 0-19 years before and after medical marijuana legalization in Massachusetts. JAMA Netw Open 2019;2:e199456.

7. Page RL, Allen LA, Kloner RA, et al. AHA scientific statement: medical marijuana, recreational cannabis, and cardiovascular health. Circulation 2020;142:e131-52.

Competing interests: None declared.

Content licence: This is an Open Access article distributed in accordance with the terms of the Creative Commons Attribution (CC BY-NC-ND 4.0) licence, which permits use, distribution and reproduction in any medium, provided that the original publication is properly cited, the use is noncommercial (i.e., research or educational use), and no modifications or adaptations are made. See: https://creativecommons.org/ licenses/by-nc-nd/4.0/ 